\title{
CARACTERIZAÇÃO ZOOSANITÁRIA DA OVINOCULTURA E DA CAPRINOCULTURA NA MICRORREGIÃO HOMOGÊNEA DE TERESINA, PIAUÍ, BRASIL
}

\author{
R.A.B. Silva ${ }^{1 *}$, M.C.S. Batista ${ }^{1 *}$, C.B. Nascimento ${ }^{1}$, R.P.A. Alves ${ }^{1}$, F.S.F. Alves $^{2}$, \\ R.R. Pinheiro ${ }^{2}$, M.S. Sousa ${ }^{1}$, B.L.M. Diniz ${ }^{1}$, J.F.S. Cardoso ${ }^{1}$, N.R.O. Paula ${ }^{1}$
}

${ }^{1}$ Universidade Federal do Piauí, Departamento de Medicina Veterinária, CP 33, CEP 64.900-000, Bom Jesus, PI, Brasil. E-mail: neyromulo@ufpi.edu.br

\section{RESUMO}

\begin{abstract}
O objetivo deste trabalho foi caracterizar o manejo sanitário e descrever as características da ovinocaprinocultura na microrregião homogênea (MRH) de Teresina, Piauí, Brasil. Foram investigadas 45 propriedades capriovinícolas, distribuídas em 14 municípios. O número médio de animais foi de 129 e 121 nos rebanhos caprinos e ovinos, respectivamente. Os animais utilizados na formação dos rebanhos base tiveram como origem os estados da Bahia, Pernambuco e Ceará. Animais SPRD (45,8\%) e da raça Santa Inês (35,4\%) são os mais encontrados entre os ovinos e na espécie caprina animais SPRD (55,3\%) e animais da raça Anglo-Nubiana (28,6\%). O estudo demostrou que $42,2 \%$ propriedades pesquisadas exploravam bovinos e caprinos e/ ou ovinos. $\mathrm{O}$ sistema de criação extensivo foi adotado em $71,1 \%$ dos criatórios. As práticas sanitárias adotadas com maior frequência foram descarte de agulhas após o uso, isolamento de animais doentes e corte e desinfecção do umbigo. A desverminação foi a prática utilizada no controle de verminoses por $100,0 \%$ dos produtores. A vacinação foi adotada em 48,8\% dos rebanhos. As alterações clínicas mais citadas foram linfadenite caseosa $(64,4 \%)$, pododermatite $(62,2 \%)$ e diarreia $(60,0 \%)$. Portanto, a ovinocaprinocultura na MRH de Teresina é praticada, em sua grande maioria, de forma extensiva e que, apesar de contar com instalações razoáveis, ainda apresenta deficiências quanto à utilização de práticas de manejo sanitário, o que acaba impossibilitando a prevenção e o controle de doenças, principalmente as de origem infecciosas e parasitárias.
\end{abstract}

PALAVRAS-CHAVE: Caracterização zoosanitária, manejo sanitário, ovinos, caprinos.

\section{ABSTRACT}

ZOOSANITARYCHARACTERIZATIONOFSHEEPANDGOATFARMINGINHOMOGENOUS MICROREGIONS OF TERESINA, PIAUI, BRAZIL. The objective of this study was to characterize the health practices and describe the characteristics of sheep and goat farming in the homogeneous microregion (HMR) in Teresina, Piaui, Brazil. Forty-five goat and sheep farms were investigated, distributed in 14 cities. The average number of animals per herd was 129 and 121, for the goats and sheep, respectively. The animals used to form the herds originated in the states of Bahia, Pernambuco and Ceará. Animals with undefined breed (45.8\%) and Santa Ines breed (35.4\%) were the most frequent among the sheep, while for the goats it was undefined breed (55.3\%) and Nubian goats $(28.6 \%)$. The study showed that $42.2 \%$ of the 45 farms surveyed raised cattle as well as goats and/or sheep. The extensive rearing system was adopted on $71.1 \%$ of the farms. The health practices adopted most often by farmers were the disposing of needles and syringes after use, isolation of sick animals, and cutting and disinfecting the navel. Deworming was a practice used to control worms by $100.0 \%$ of producers. Vaccination was a practice adopted in $48.8 \%$ of the herds. The clinical changes most often mentioned were caseous lymphadenitis (64.4\%), foot rot $(62.2 \%)$ and diarrhea $(60.0 \%)$. In conclusion, sheep and goat farming in the Teresina HMR is practiced extensively with reasonably proper installations, but still shows deficiencies in the use of health practices, which impair the prevention and control of diseases, particularly infectious and parasitic diseases.

KEY WORDS: Zoosanitary characterization, health practices, sheep, goats.

${ }^{2}$ EMBRAPA Caprinos e Ovinos, Sobral, CE, Brasil.

*Programa de Pós-Graduação em Ciência Animal-UFPI, Teresina, PI, Brasil. 
No cenário nacional, o Estado do Piauí ocupa o terceiro lugar na criação de caprinos, com um rebanho efetivo da ordem de 1,389 milhões, ficando aquém dos estados da Bahia e Pernambuco. Quanto à ovinocultura, apresenta-se com aproximadamente 1,387 milhões de cabeças, produção inferior aos Estados da Bahia, Ceará e Pernambuco (IвGe, 2009).

O desenvolvimento da ovinocultura e da caprinocultura no Nordeste é severamente afetado por inúmeros fatores, dentre os quais, práticas de manejo inadequadas, especialmente sanitárias, representadas pela falta de higiene das instalações e falhas na aplicação de vermífugos e vacinas (CAldas, 1989; Souza Neto et al., 1996), que acabam refletindo nas altas incidências de doenças carenciais, parasitárias e infecto-contagiosas e, dessa forma, determinando a baixa produtividade no setor (PinHeiro et al., 2000).

Os baixos índices de aplicação de práticas de manejo sanitário encontrados em propriedades do Nordeste, sem dúvida, contribuem para a manutenção dos altos níveis de morbidade e de mortalidade (Pinheiro et al., 2000; De Boer et al., 1986), que acabam se tornando a principal causa das baixas taxas de desfrute por parte dos criadores nesta região (Figueira et al., 2009).

A obtenção de dados referentes aos aspectos epidemiológicos/zoosanitários da ovinocultura e da caprinocultura regionais é fundamental para a implementação de políticas públicas formuladas especificamente para esse segmento. Considerando a carência de dados referentes à caprinovinocultura no Piauí e ressaltando-se as espécies caprina e ovina como as que melhor se adaptam às condições edafoclimáticas locais, este trabalho objetivou descrever a situação atual observada nas explorações de caprinos e ovinos na MRH de Teresina, Piauí, Brasil.

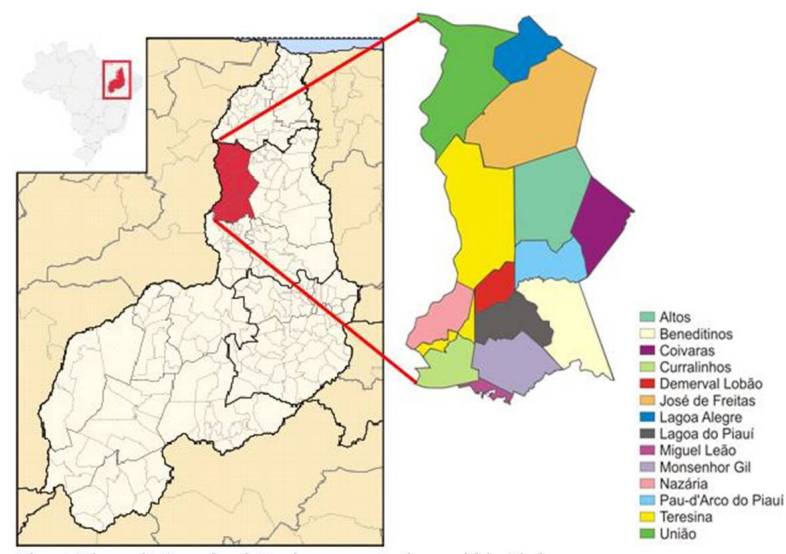

Fig. 1-Microrregião homogênea de Teresina com os respectivos municípios, Piauí. Fonte: WIKIPÉDIA (2008): Ibge, 2009.
O estudo foi conduzido em 45 unidades produtoras de caprinos e/ou ovinos georeferenciadas, distribuídas na MRH de Teresina, Piauí (Figs. 1 e 2). A MRH de Teresina está situada na mesorregião centro-norte piauiense, possui localização entre as longitudes $42^{\circ} 48^{\prime} \mathrm{W}$ e $42^{\circ} 34^{\prime} \mathrm{W}$ e latitude entre $4^{\circ}$ $45^{\prime} \mathrm{S}$ e $5^{\circ} 40^{\prime} \mathrm{S}$.

O levantamento de dados foi realizado no período de junho a dezembro de 2010, quando foram aplicados questionários investigativos adaptados por BANDEIRA (2005) em 43 rebanhos de caprinos e 23 de ovinos, uma vez que nem sempre foi possível aplicar o questionário em propriedades com as duas explorações pecuárias. Com base nos questionários aplicados, foi investigado o perfil produtivo, as características das instalações e de manejo sanitário, bem como os sistemas de criação, como descritos nas Tabelas 1, 2, 3, 4, 5, 6 e 7. O sistema de criação foi identificado de acordo com a descrição de MourA SOBRINHO (2008).

O delineamento amostral foi dividido em dois estágios. O primeiro constituiu-se na obtenção das unidades primárias da amostra, ou seja, o número de rebanhos pertencentes à MRH Teresina que participariam do estudo. A escolha da unidade primária de amostragem foi baseada no cadastro das propriedades rurais, produtoras de caprinos e ovinos, da Agência de Defesa Agropecuária do Estado do Piauí (ADAPI), através de sorteio aleatório. Se uma propriedade não pôde, por qualquer motivo, ser visitada, ela foi substituída por outra, nas proximidades e com as mesmas características de produção. Já no segundo estágio, foi determinado o número mínimo de rebanhos por município (unidades secundárias de amostra), por meio de estratificação, baseada no rebanho efetivo de cada município cadastrado na ADAPI.

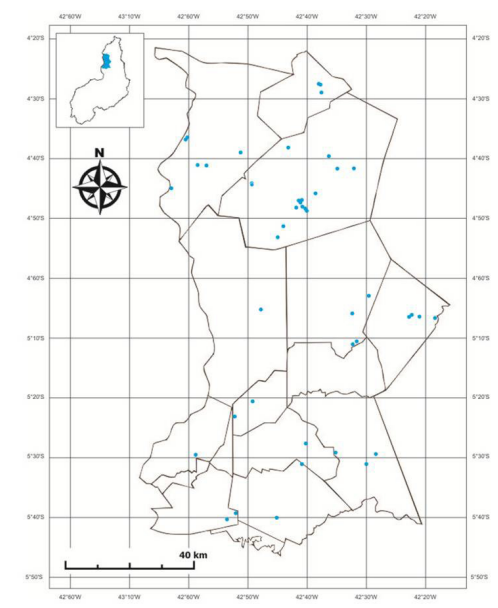

Fig. 2 - Distribuição geográfica das propriedades georeferenciadas analisadas na Microrregião Homogênea de Teresina, Piauí, Brasil. 
As informações obtidas por meio dos questionários aplicados aos produtores foram usadas para elaboração de um banco de dados, que foi analisado com auxílio do programa Epi-info 3.2.2 (DeAn et al., 1992).

Podemos verificar, na Figura 2, a distribuição espacial georeferenciada, relativa às propriedades analisados para a sorologia para o Lentivírus de Pequenos Ruminantes (LVPR) na Microrregião Homogênea de Teresina, Piauí, Brasil. Nas propriedades pesquisadas o número de animais no rebanhocaprino variou de 12 a 1.000, com média de 129 animais, número superior aos 79 animais relatados no Estado do Tocantins por Moura Sobrinho (2008) e aos 114 encontrados por PINHeIro et al. (2000) no Estado do Ceará. Quanto ao rebanho ovino, o número médio de animais encontrado nesse estudo foi de 121, com rebanhos possuindo de 23 a 700 animais, resultado superior à média de 54 encontrada por SouZA Neto et al. (1995), no Estado do Piauí, e bem abaixo da média de 340 encontrada por Moura SOBRINHO (2008) no Estado de Tocantins. Essas diferenças podem ser explicadas pela confirmação do último senso realizado pelo IBGE (2009), onde se pode verificar um aumento considerável do rebanho ovino no Estado de Tocantins.

De acordo com a avaliação dos questionários, foi possível observar que, para formar os rebanhos base da MRH de Teresina, Piauí, 93\% das criações de caprinos e ovinos utilizaram animais provenientes do próprio estado. Outros estados citados foram Bahia, Ceará, Pernambuco, Paraíba e Rio Grande do Sul. Em 13,3\% dos casos, era comum a aquisição dos animais de dois ou mais estados (Tabela 1). Esses dados são relevantes para traçar possíveis medidas de análise de risco posteriormente, frente aos órgãos de fiscalização agropecuária, tendo como base o rastreamento dos animais envolvidos.

Dentre os criadores de ovinos, animais sem padrão racial definido (SPRD), das raças Santa Inês, Dorper e Morada Nova foram utilizados,

Tabela 1 - Distribuição de frequência da origem dos rebanhos base para formação dos criatórios de caprinos e ovinos na MRH de Teresina, Piauí, 2010.

\begin{tabular}{lcc}
\hline \multirow{2}{*}{ Estado } & \multicolumn{2}{c}{$\%$} \\
\cline { 2 - 3 } & Caprino & Ovino \\
\hline Piauí & 93,1 & 93,3 \\
Bahia & 9,1 & 10,0 \\
Pernambuco & 9,1 & 6,7 \\
Ceará & 6,8 & 6,7 \\
Paraíba & 2,3 & 3,3 \\
Rio Grande do Sul & 2,3 & 3,3 \\
\hline
\end{tabular}

respectivamente, por $73,3 \%, 56,7 \%, 26,7 \%$ e $3,3 \%$. Quanto aos animais da espécie caprina, em 70,4\% dos animais foram considerados SPRD, 36,4\% dos rebanhos possuiam animais da raça Anglo-Nubiana, $15,9 \%$ da raça Boer e 2,3\% animais da raça Savana e Canindé. A criação de animais de pelo menos duas raças foi uma característica comum observada entre os criadores de animais de elite. Nesse contexto, podemos observar uma certa preferência para a criação de determinadas raças por parte dos criadores nesta região, o que pode viabilizar o direcionamento de pesquisas que visem o incremento produtivo desses rebanhos.

Com relação ao sistema de produção, o sistema extensivo foi praticado por $71,1 \%$ dos criatórios de caprinos e ovinos dessa MRH, o sistema semiintensivo foi utilizado por $24,4 \%$ dos produtores e somente $4,5 \%$ adotavam o sistema intensivo (Tabela 2). Esses resultados se assemelham aos encontrados por PinHeIro et al. (2000), em rebanhos caprinícolas do Estado do Ceará, onde, assim como no presente estudo, na maioria das propriedades os animais eram criados em regime extensivo $(77,9 \%)$, enquanto o sistema de criação semi-intensivo era adotado em $19,6 \%$ e a criação de forma intensiva em apenas $1,6 \%$. Isto vem demonstrando que o Nordeste do Brasil vem, ainda, mantendo determinados padrões rudimentares na criação de pequenos ruminantes, o que reflete na baixa produtivodade dos mesmos.

Das criações de ovinos avaliadas, as que recebiam algum tipo de assistência técnica somaram 52,9\%, já entre as criações de caprinos esse tipo de benefício esteve presente somente em 47,7\% (Tabela 3). Esses resultados foram inferiores aos observados por Moura Sobrinho (2008) no Estado do Tocantins e por BANDEIRA et al. (2005) em propriedades da microrregião do Cariri ocidental e oriental do Estado da Paraíba, onde 93,3\% dos produtores de caprinos recebiam algum tipo de assistência técnica. Esse critério de assistencialismo está diretamento ligado ao sucesso da produção pecuária, uma vez que a prática de medidas básicas em um criatório poderá elevar os rendimentos e a melhorar a vida dos pequenos criadores da região.

Tabela 2 - Distribuição de frequência de sistema de criação de rebanhos ovicaprinícolas na MRH de Teresina, Piauí, 2010.

\begin{tabular}{lcc}
\hline Sistema de criação & $\mathrm{n} / \mathrm{N}$ & Frequência \% \\
\hline Semi-intensivo & 11 & 25,4 \\
Intensivo & 2 & 4,5 \\
Extensivo & 32 & 71,1 \\
\hline Total & 45 & 100,0 \\
\hline
\end{tabular}


As práticas sanitárias adotadas com maior frequência pelos criadores foram: o descarte de agulhas e seringas após o uso $(75,5 \%)$, o isolamento dos animais doentes $(71,1 \%)$ e o corte e desinfecção do umbigo (53,3\%) (Tabela4). Quantoàs praticas decontrolecontra verminoses, as mais citadas foram a desverminação $(100,0 \%)$, a alternância anual do vermífugo $(75,5 \%)$ e a desverminação de animais recém-chegados $(64,4 \%)$ (Tabela 5). A desverminação foi a prática adotada com maior frequência, variando deuma seis vezes porano. Esses resultados foram semelhantes aos encontrados no Estado de São Paulo, na região de Araçatuba, por NogueIRA et al. (2007) que relataram a adoção desta prática por $100 \%$ dos ovinocultores e se assemelham aosencontrados por PINHEIROetal. (2000), querelataram 95\% de criatórios caprinícolas no estado do Ceará e aos resultados de SouZA NeTo (1987) que verificou uma frequência de $96 \%$ em rebanhos caprinos de leite no Estado do Pernambuco. Alencaret al. (2010) relataram 88,2\% de desverminaçãoem rebanhoscaprinoseovinos do sertão do Estado de Pernambucano e, de acordo com RodRigues et al. (2005), 77\% dos caprinocultores do Sudoeste desse estado adotavam essa prática de manejo sanitário. Apesar de a desverminação ser uma prática bem adotada entre os criadores entrevistados, ressalta-se que se esse manejo for feito de forma indiscriminada, sem acompanhamento técnico, poderá inviabilizar a produção dos rebanhos que realizam tal medida, podendo induzir a resistência parasitária aos medicamentos utilizados.

A ivermectina foi o princípio ativo utilizado em $79,3 \%$ das propriedades. A desverminação dos animais recém-chegados foi praticada em $64,4 \%$ dos rebanhos, resultado superior ao relatado por ALENCAR et al. (2010), que encontraram $51,7 \%$ de criadores que adotavam essa prática no sertão do Estado de Pernambucano. A realização de quarentena nos animais recém-chegados foi a prática menos adotada $(8,8 \%)$ verificada neste estudo. A adoção dessa prática pode ser considerada como um dos principais meios para se evitar a introdução e disseminação de doenças nos rebanhos (PINHeIro et al., 2000).

Tabela 3 - Distribuição de frequência dos profissionais, periodicidade e tipo de assistência técnica em criatórios de caprinos e ovinos na MRH de Teresina, Piauí, 2010.

\begin{tabular}{|c|c|c|c|c|}
\hline \multirow{2}{*}{ Assistência técnica } & \multicolumn{2}{|c|}{ Caprino } & \multicolumn{2}{|c|}{ Ovino } \\
\hline & $\mathrm{N} / \mathrm{n}$ & $\%$ & $\mathrm{~N} / \mathrm{n}$ & $\%$ \\
\hline Sim & $21 / 44$ & 47,7 & $18 / 34$ & 52,9 \\
\hline Não & $23 / 44$ & 52,3 & $16 / 34$ & 47,1 \\
\hline \multicolumn{5}{|l|}{ Profissional } \\
\hline Médico veterinário & $19 / 21$ & 90,5 & $17 / 34$ & 94,4 \\
\hline Zootecnista & - & - & - & - \\
\hline Engenheiro agrônomo & - & - & - & - \\
\hline Técnico agropecuário & $2 / 21$ & 9,5 & $1 / 18$ & 5,6 \\
\hline \multicolumn{5}{|l|}{ Periodicidade } \\
\hline Semanal & $5 / 21$ & 23,9 & $4 / 18$ & 22,2 \\
\hline Quinzenal & $2 / 21$ & 9,5 & $2 / 18$ & 11,1 \\
\hline Mensal & $4 / 21$ & 19,0 & $3 / 18$ & 16,7 \\
\hline Semestral & $2 / 21$ & 9,5 & $2 / 18$ & 11,1 \\
\hline Quando precisa & $7 / 21$ & 38,1 & $7 / 18$ & \\
\hline \multicolumn{5}{|l|}{ Tipo de assitência } \\
\hline Pública & - & - & - & \\
\hline Privada & $21 / 21$ & 100,0 & $18 / 18$ & \\
\hline
\end{tabular}

Tabela 4 - Distribuição de frequência das práticas sanitárias adotadas nos criatórios de caprinos e ovinos na MRH de Teresina, Piauí, 2010.

\begin{tabular}{lcc}
\hline Prática & $\mathrm{n} / \mathrm{N}$ & Frequência \% \\
\hline Descarte de agulhas após o uso & $34 / 45$ & 75,5 \\
Isolamento de animais doentes & $32 / 45$ & 71,1 \\
Corte e desinfecção do umbigo & $24 / 45$ & 53,3 \\
Vacinação & $22 / 45$ & 48,8 \\
Entera ou crema animais mortos & $20 / 45$ & 44,4 \\
Separação de machos e fêmeas & $14 / 45$ & 31,1 \\
Utilização de piquete maternidade & $13 / 45$ & 28,8 \\
Descanso de pastagem & $12 / 45$ & 26,6 \\
Realização de quarentenário & $4 / 45$ & 8,8 \\
\hline
\end{tabular}


Tabela 5 - Distribuição de frequência das práticas de controle contra verminoses adotadas nos criatórios de caprinos e ovinos na MRH de Teresina, Piauí, 2010.

\begin{tabular}{lcc}
\hline Prática & $\mathrm{n} / \mathrm{N}$ & Frequência $\%$ \\
\hline Desverminação & $45 / 45$ & 100,0 \\
Alternância anual do vermífugo & $34 / 45$ & 75,5 \\
Desvermina animais recém-chegados & $29 / 45$ & 64,4 \\
Uso de esterqueira & $13 / 45$ & 28,8 \\
Permanência noaprisco de12horas apósa desverminação & $11 / 45$ & 24,4 \\
\hline
\end{tabular}

Tabela 6 - Distribuição da frequência de vacinação e do tipo de vacinação em criatórios da MRH de Teresina, Piauí, 2010.

\begin{tabular}{lcc}
\hline Vacinação dos animais & $\mathrm{n} / \mathrm{N}$ & Frequência \% \\
\hline Sim & $22 / 45$ & 48,9 \\
Não & $23 / 45$ & 51,1 \\
\hline Tipo de vacina & $\mathrm{n} / \mathrm{N}$ & Frequência $\%$ \\
\hline Clostridioses & $15 / 22$ & 68,2 \\
Raiva & $4 / 22$ & 18,2 \\
Clostridioses + Raiva & $2 / 22$ & 9,1 \\
Clostridioses + Raiva + Linfadenite & $1 / 22$ & 4,5 \\
\hline
\end{tabular}

A imunoprofilaxia contra clostridioses, raiva e linfadenite caseosa foram utilizadas entre os criadores entrevistados. Os criadores que vacinavam seus animais contra pelo menos uma doença somaram $48,9 \%$, sendo mais frequente a imunoprofilaxia contra clostridioses $(81,8 \%)$. Resultado esses que se assemelham aos obtidos por BANDEIRA (2005) e por ALENCAR et al. (2010) que encontraram um percentual elevado de utilização da vacina contra clostridioses. Das propriedades que aplicam vacinas, $86,4 \%$ administram somente contra uma das doenças citadas, $9,1 \%$ contra duas e $4,5 \%$ contra três (Tabela 6).

Pela a análise dos questionários epidemiológicos/ zoosanitários, abscessos subcutâneos sugestivos de linfadenite caseosa apresentam-se como o problema mais frequente, registrado em $64,4 \%$ dos rebanhos. Abscessos subcutâneos foram as alterações clínicas mais comuns em rebanhos caprinos do Estado do Pernambuco (Souza Neto, 1987; Alencaretal., 2010). A pododermatite apresentou uma frequência de $62,2 \%$ e a diarreia foi um problema relatado por $60 \%$ dos criadores neste estudo (Tabela 7).

Em relação às LVPR, 28,8\% dos criadores entrevistados tinham conhecimento dessas enfermidades e somente 15,5\% utilizavam diagnóstico sorológico, sendo que desses, dois criadores associavam o diagnóstico clínico ao laboratorial. Vale ressaltar que a prática do diagnóstico contra lentiviroses foi adotada em $100 \%$ dos criadores de animais de elite que participaram desseestudo, ao passo que nenhum dos criadores de animais de rebanho geral (SPRD) adotou qualquer tipo de método diagnóstico.

A análise dos dados demonstrou a presença, na MRH de Teresina, Piauí, Brasil, de uma atividade onde predomina o sistema de criação extensivo e que ainda apresenta deficiências quanto à prática e adoção de medidas de manejo sanitário.

Tabela 7 - Distribuição de frequência dos sinais clínicos e doenças de acordo com 45 produtores de caprinos e ovinos da MRH de Teresina, Piauí, 2010.

\begin{tabular}{lcc}
\hline Sinais clínicos/doenças & $\mathrm{n} / \mathrm{N}$ & Frequência \% \\
\hline Abscessos cutâneos & $29 / 45$ & 64,4 \\
Pododermatite & $28 / 45$ & 62,2 \\
Diarréia & $27 / 45$ & 60,0 \\
Miíase/bicheira cutânea & $22 / 45$ & 48,8 \\
Mamite & $13 / 45$ & 28,8 \\
Ectoparasitas & $12 / 45$ & 26,2 \\
Pneumonia & $10 / 45$ & 22,2 \\
Ectima contagioso & $5 / 45$ & 11,1 \\
Aborto & $4 / 45$ & 8,8 \\
Ceratoconjuntivite & $3 / 45$ & 6,6 \\
Alterações nervosas & $3 / 45$ & 6,6 \\
Artrite & $2 / 45$ & 4,4 \\
\hline
\end{tabular}

A MRH Teresina, Piauí apresenta um grande potencial na produção de caprinos e ovinos, porém esse segmento ainda carece de profissionalização, da implementação de assistência técnica por meio de políticas públicas, o que se traduzirá em forte impacto na economia estadual e melhorias na qualidade de vida dos caprinovinocultorees da região.

Agradecemos à Coordenação de Aperfeiçoamento de Pessoal de Nível Superior (CAPES), pelo suporte financeiro através da concessão de bolsa de estudos; eà Empresa Brasileira de Pesquisa Agropecuária (EMBRAPA) Centro Nacional de Pesquisa de Caprinos e Ovinos, por viabilizar a conclusão desse trabalho, através do projeto aprovado pelo MAPA/ CNPq, edital 64/2009. 


\section{REFERÊNCIAS}

ALENCAR, S.P.; MOTA, R.A.; COELHO, M.C.O.C.; NASCIMENTO, S.A.; BREU, S.R.O.; CASTRO, R.S. Perfil sanitário dos rebanhos caprinos e ovinos no sertão de Pernambuco. Ciência Animal Brasileira, v.11, n.1, p.131-140, 2010.

BANDEIRA, D.A. Características sanitárias e de produção da caprinocultura nas microrregiões do Cariri do Estado da Paraíba. 2005. 117f. Tese (Doutorado em Ciência Veterinária) - Universidade Federal Rural de Pernambuco, Recife, 2005.

CALDAS, E.M. Estudo da ovinocaprinocultura na região nordeste do Estado da Bahia. Arquivos da Escola de Medicina Veterinária da UFBA, v.12, n.1, p.1-98, 1989.

DE BOER, A.J., GUTIERREZ, A., SOUZA NETO, J. Farm-level resources for small ruminant production. In: REUNIÃO TÉCNICO-CIENTÍFICA DO PROGRAMA DE APOIO À PESQUISA COLABORATIVA DE PEQUENOS RUMINANTES, 1986. Sobral, Anais. Sobral: EMBRAPA, 1986. p.9-36.

DEAN, A.G.; DEAN, J.A.; BURTON, A.H.E.; DICKER R.C. Epi info, version 6: a Word processing, database and statistic program for epidemiology on microcomputers. Atlanta, Georgia, Center for Disease Conrol, 1992. 302p. Disponível em: <http://www.cdc.gov/ Epilnfo/biblio.htm>. Acesso em: 20 dez. 2010.

FILGUEIRA, T.M.B. AHIB, S.M.M.; SUASSUNA, A.C.D.; SOUZA, W.J.; FONSECA, Z.A.A.S. Aspectos epidemiológicos e sanitários das criações de caprinos na região da chapada do Apodi. Revista Verde, v.4, n.2, p.64-67, 2009.

IBGE - INSTITUTO BRASILEIRO DE GEOGRAFIA E ESTATÍSTICA. Produção da Pecuária Municipal 2009. Rio de Janeiro, RJ, 2010. Disponível em: <http://www.sidra.ibge.gov.br/bda/tabela/listabl. asp?c=73\&z=p\&o=23>. Acesso em: 10 out. 2010.

MOURA SOBRINHO, P.A. Características de produção da ovinocaprinocultura e soro prevalência de lentiviroses de pequenos ruminantes no Estado de Tocantins. 2008. $119 \mathrm{f}$. Tese (Doutorado em Ciência Veterinária) - Universidade Federal Rural de Pernambuco, Recife, 2008.

NOGUEIRA, A.H.C.; CURCI, V.C.L.M.; FERRARI, C.I.L.; CARDOSO, T.C. Aspectos epidemiológicos da ovinocultura na região de Araçatuba: dados preliminares. Biológico, São Paulo, v.68, p.33, 2007. Suplemento. Disponível em: <http:/ /www.biologico.sp.gov.br/ biologico/v68_supl_raib/033.pdf>. Acesso em: $21 \mathrm{fev}$. 2008.

PINHEIRO, R.R.; GOUVEIA, A.M.G.; ALVES, F.S.F.; HADDAD, J.P.A. Aspectos epidemiológicos da caprinocultura cearense. Arquivo Brasileiro de Medicina Veterinária e Zootecnia, v.52, n.5, p.534-543, 2000.

RODRIGUES, C.F.C.; MELLO, N.T.C.; LEINZ, F.F.; CARVALHO FILHO, A.C.; BIANCHINI, D.; SANNAZZARO, A.M. Aspectos sanitários da caprinocultura familiar na região Sudoeste paulista. São Paulo. Arquivos do Instituto Biológico, São Paulo, v.72, p.1-64, 2005. Suplemento 2 .

SOUZA NETO, J. Características gerais da caprinocultura leiteira no estado de Pernambuco. Sobral: EMBRAPA CNPC, 1987. (Boletim n. 4).

SOUZA NETO, J.; BAKER, G.A.; SOUSA, F.B. Análise socioeconômica da exploração de caprinos e ovinos no Estado do Piauí. Pesquisa Agropecuária Brasileira, v.30, n.8, p.1017-1030, 1995.

SOUZA NETO, J.; BAKER, G.A.; SOUSA, F.B. Caprinocultura de duplo propósito no Nordeste do Brasil: avaliação do potencial produtivo Sobral - CE, Embrapa/CNPC, 1996. p.210-212. (. Relatório Técnico do Centro Nacional de Pesquisa de Caprinos, 1987-1995).

WIKIPÉDIA. Geografia do Piauí, 2008. Disponivel em: <http://pt.wikipedia.org/wiki/Microrregi\%C3\% A3o_de_ Teresina>. Acesso em: 23 jan. 2010.

Recebido em 13/4/11

Aceito em 3/11/11 